\title{
Observaciones sobre la ecología poblacional del ratón doméstico en Lachay, Perú
}

Observations sur l'écologie des populations de souris domestiques de Lachay, Pérou

Notes on the population ecology of the domestic mouse in Lachay, Peru

César Kunimoto, Cecilia De la Cruz, Margarita Arana y Oswaldo E. Ramírez

\section{(2) OpenEdition}

Journals

\section{Edición electrónica}

URL: http://journals.openedition.org/bifea/6753

DOI: $10.4000 /$ bifea.6753

ISSN: 2076-5827

\section{Editor}

Institut Français d'Études Andines

\section{Edición impresa}

Fecha de publicación: 1 agosto 2002

Paginación: 323-328

ISSN: 0303-7495

\section{Referencia electrónica}

César Kunimoto, Cecilia De la Cruz, Margarita Arana y Oswaldo E. Ramírez, « Observaciones sobre la ecología poblacional del ratón doméstico en Lachay, Perú », Bulletin de l'Institut français d'études andines [En línea], 31 (2) | 2002, Publicado el 08 agosto 2002, consultado el 07 diciembre 2020. URL http://journals.openedition.org/bifea/6753 ; DOl : https://doi.org/10.4000/bifea.6753

\section{(ब) $\Theta \Theta$}

Les contenus du Bulletin de l'Institut français d'études andines sont mis à disposition selon les termes de la licence Creative Commons Attribution - Pas d'Utilisation Commerciale - Pas de Modification 4.0 International. 


\title{
OBSERVACIONES SOBRE LA ECOLOGÍA POBLACIONAL DEL RATÓN DOMÉSTICO EN LACHAY, PERÚ
}

\author{
César KUNIMOTO, Cecilia DE LA CRUZ, Margarita ARANA, \\ Oswaldo E. RAMIREZ
}

\section{Resumen}

Se estudiaron algunos aspectos de la ecología poblacional del ratón doméstico (Mus musculus) durante el inicio y transcurso de la estación seca en las lomas de Lachay. La abundancia poblacional del ratón doméstico disminuyó drásticamente en los tres sectores estudiados a diferentes altitudes durante la estación seca. Las proporciones de sexo mostraron que el número de machos fue siempre mayor al número de hembras. El ámbito de hogar no presentó diferencias estadísticamente significativas $(p<0,05)$ en los tres sectores estudiados. Nuestros resultados sugieren que la abundancia de plantas es probablemente uno de los factores más importantes en la regulación de la población de esta especie. Además, se formula la hipótesis que las hembras son más sensibles que los machos a las difíciles condiciones climáticas de la estación seca.

Palabras claves: Ecología, ratón doméstico, Mus musculus, lomas, Perú.

\section{OBSERVATIONS SUR L'ÉCOLOGIE DES POPULATIONS DE SOURIS DOMESTIQUES DE LACHAY, PÉROU}

\section{Résumé}

L'étude de l'écologie des populations de souris domestiques (Mus musculus) des "lomas de Lachay" met en évidence leur réaction aux variations climatiques et florales au début et pendant la saison sèche. Dans les trois secteurs étudiés, situés à des altitudes différentes, la densité de population de cette espèce diminue d'une façon très importante durant la saison sèche. Les mâles sont toujours plus nombreux que les femelles. La distribution des individus dans l'espace ne montre pas de différences significatives entre les trois secteurs. Ces résultats suggèrent que l'abondance de la végétation est probablement un des facteurs les plus importants dans la régulation de la densité de population de cette espèce. De plus, les femelles semblent plus sensibles que les mâles aux conditions climatiques difficiles de la saison sèche.

Mots clés : Écologie, souris domestiques, Mus musculus, lomas, Pérou.

"Departamento de Ciencias Biológicas y Fisiológicas, Facultad de Ciencias y Filosofía, Universidad Cayetano Heredia. Av. Honorio Delgado No 430, San Martín de Porres, Lima - Perú. Telefax: (51 1) 31900 19. E-mail: oramirez@upch.edu.pe 


\title{
NOTES ON THE POPULATION ECOLOGY OF THE DOMESTIC MOUSE IN LACHAY, PERU
}

\begin{abstract}
In this article we present some aspects related to the population ecology of Mus musculus at the beginning and throughout the course of the dry season in the Lachay lomas. Population abundance in three zones with different altitudes decreased drastically during the dry season. Sex proportions showed that the number of males was always higher than the number of females. Home range was not statistically different between the three analyzed areas. Our results suggest that the presence and/or abundance of plants may be one of the most important in regulating the population this species. In addition our study suggests the hypothesis that females of Mus musculus are more sensitive tha males to the adverse climatic conditions in the Lachay National Reserve during dry season.
\end{abstract}

Key words: Ecology, domestic mouse, Mus musculus, lomas, Peru.

\section{INTRODUCCIÓN}

El ecosistema de lomas es una formación vegetal altamente estacional que se establece como consecuencia de la condensación de la neblina sobre los primeros contrafuertes de la Cordillera de los Andes en los desiertos costeros de Perú y norte de Chile (Ferreyra, 1987; Dillon, 1997). Las lomas de Lachay se localizan en la costa central del Perú, a $105 \mathrm{~km}$ de Lima, presentando las zonas de vida conocidas como Desierto Montano Bajo Subtropical y Desierto Perárido Subtropical (INRENA, 1994).

Como cualquier especie introducida, el ratón doméstico (Mus musculus, L.) ha tenido que enfrentar un proceso de aclimatación y/o adaptación a sus nuevos ambientes, particularmente en aquellos casos donde las condiciones climatológicas determinan ambientes extremos, como es el caso de los desiertos. La alta estacionalidad de las lomas en los desiertos peruanos exige que sus residentes aprovechen al máximo la abundancia de los recursos durante la estación húmeda y que resistan las desfavorables condiciones bióticas y abióticas de la estación seca. El ratón doméstico europeo, introducido por los conquistadores españoles hace un poco más de 500 años, es una de las especies de mamífero más exitosas en términos de abundancia en la Reserva Nacional de Lachay. El estudio de su dinámica poblacional en un ambiente altamente estacional permitirá entender los aspectos básicos de sus estrategias poblacionales para alcanzar su éxito ecológico en los ambientes de lomas.

En el presente artículo presentamos el primer estudio sobre ecología poblacional del ratón doméstico en el Perú. Durante el período de estudio (diciembre-junio,1998/ 1999) se incluye el inicio, desarrollo y finalización de la estación seca. Debido a que la altitud es uno de los factores más importantes para la condensación de la neblina, principal fuente de humedad y agua en este ecosistema, el estudio contiene una evaluación en tres sectores altitudinales de las lomas de Lachay.

\section{MATERIAL Y MÉTODOS}

El trabajo de campo fue realizado en la quebrada Teatinos, un sector localizado en la parte

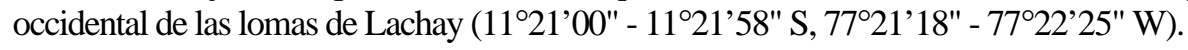


A lo largo de la quebrada Teatinos se construyeron tres cuadrantes a diferentes altitudes (280, 310 y $450 \mathrm{~m}$ sobre el nivel del mar). Cada cuadrante estuvo constituido por 45 estaciones de colecta distribuidas en un área de 40 × 20 m (5 m de separación entre cada estación), los cuales fueron evaluados por lo menos dos noches consecutivas cada quince días. Durante los días de evaluación de los cuadrantes, en cada estación de colecta se colocó una trampa para captura viva (alrededor de las 5 p.m.), la cual fue cebada con una mezcla de avena, mantequilla de maní y vainilla. Se revisaron las trampas al día siguiente (alrededor de las 7 a.m.) y los animales colectados fueron marcados (por amputación de las falanges) y liberados en los puntos de captura. Para cada animal colectado se registró el sexo y lugar de captura. La abundancia relativa de la población fue determinada en base al número de animales capturados en 45 trampas por noche para cada cuadrante independientemente. Las estimaciones de la abundancia relativa se realizaron quincenalmente entre diciembre de 1998 y junio de 1999.

Los especímenes colectados en los tres cuadrantes de la quebrada Teatinos fueron utilizados para estimar la proporción de sexos. El sexo de los animales capturados fue determinado por el examen de los genitales externos. El ámbito de hogar fue calculado utilizando los métodos del polígono mínimo convexo (Mohr, 1947) y la media harmónica (Dixon \& Chapman, 1980) utilizando el programa CALHOME v 1.0 (Home Range Analysis Program, Kie, et al., 1994). Sólo los animales con al menos cuatro capturas fueron incluidos en los análisis del ámbito de hogar. Para analizar si existen diferencias estadísticamente significativas se utilizó la prueba no paramétrica de Kruskal-Wallis con un nivel de significación de $\mathrm{p}<0,05$.

\section{RESULTADOS}

Las variaciones en la abundancia poblacional de Mus musculus se muestran en la figura 1. Los tres sectores altitudinales que fueron analizados, alto $(450 \mathrm{~m})$, medio (310 $\mathrm{m}$ ) y bajo $(280 \mathrm{~m})$, presentaron sus valores más altos en el mes de diciembre. Con el transcurso de la estación seca (entre diciembre y marzo) la abundancia poblacional disminuyó progresivamente hasta alcanzar los niveles más bajos entre marzo y junio, antes del inicio de la estación húmeda (julio a octubre). Existieron diferencias marcadas en el comportamiento de las curvas poblacionales en función de su ubicación altitudinal. Durante todo el período de estudio, se registraron los más altos valores de abundancia en el cuadrante alto.

Las proporciones sexuales se calcularon a partir de 243 animales capturados en los tres cuadrantes. La proporción machos:hembras fue 1,5:1 en el cuadrante alto, 7:1 en el cuadrante medio y 2,8:1 en el cuadrante bajo. La proporción machos:hembras total (incluyendo los animales de los tres cuadrantes) fue 2,1:1.

El ámbito de hogar del ratón doméstico, utilizando los métodos del polígono convexo mínimo y la media harmónica respectivamente, fue: $142 \mathrm{~m}^{2}$ y $129 \mathrm{~m}^{2}$ en el cuadrante bajo ( $\mathrm{n}=3$ individuos), $100 \mathrm{~m}^{2}$ y $82 \mathrm{~m}^{2}$ en el cuadrante medio $(\mathrm{n}=1$ individuo), $50 \mathrm{~m}^{2}$ y $51 \mathrm{~m}^{2}$ en el cuadrante alto $(\mathrm{n}=4$ individuos). Las diferencias en el ámbito de hogar entre los tres cuadrantes no fueron estadísticamente significativas. 


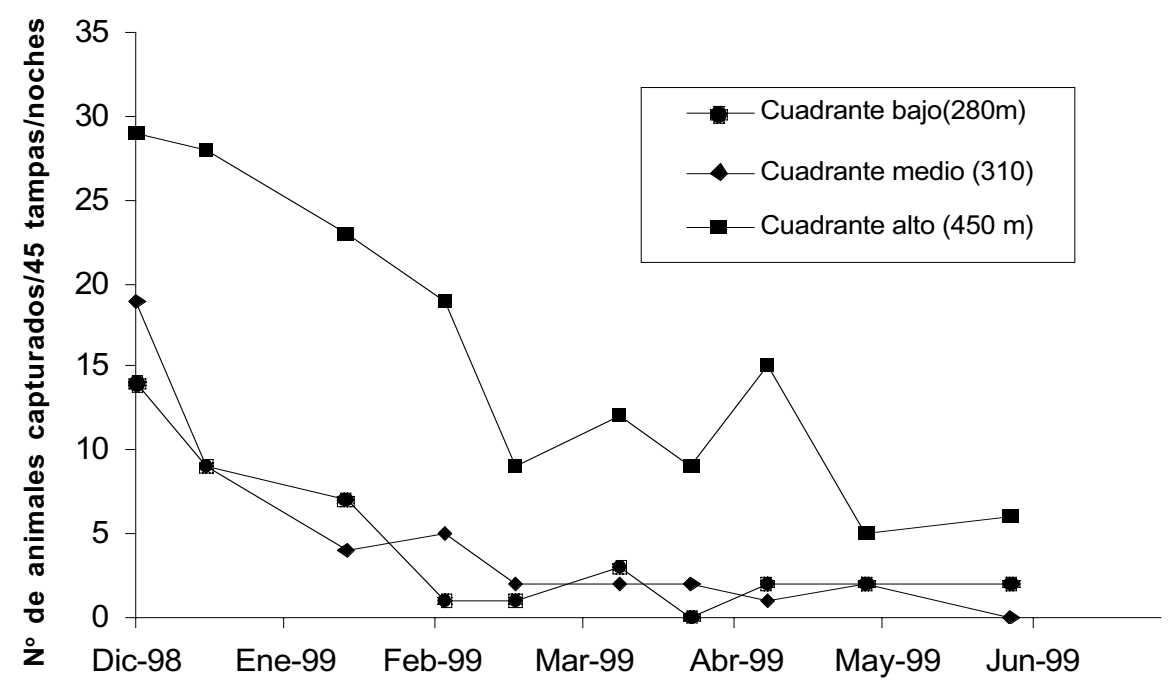

Fig. 1 - Abundancia poblacional de Mus musculus en los tres cuadrantes de la quebrada Teatinos durante la estación seca (98-99), Reserva Nacional de Lachay, Perú.

\section{DISCUSIÓN}

La drástica reducción en la abundancia poblacional del ratón doméstico entre los meses de diciembre y febrero muestra una interesante correspondencia con los característicos cambios de temperatura y humedad al inicio y desarrollo de la época seca en las lomas de Lachay (Fig. 2). Durante este período, cuando la temperatura es alta y la humedad relativa baja, la mayor parte de la vegetación de los cuadrantes se seca, permaneciendo sólo algunos árboles y arbustos especializados en tolerar las difíciles condiciones de la estación seca. Sin embargo, las zonas más altas presentan una humedad relativa mayor que las zonas bajas, por lo que las zonas altas son los últimos sectores de las lomas en sufrir la desecación y muerte de la vegetación. Debido a que en el presente estudio no se ha realizado una evaluación cuantitativa de la profusión vegetal, no es posible realizar una correlación entre las abundancias de vegetación y ratón doméstico. No obstante, nuestros resultados sugieren que la mayor cantidad de ratón doméstico en el cuadrante alto está directamente relacionado con la mayor permanencia de la vegetación en las zonas altas de las lomas. Entre los meses de marzo y junio, aunque la temperatura disminuye y la humedad incrementa, los niveles de retención de agua en el suelo no son suficientemente altos como para permitir la germinación de las semillas, la cual ocurre usualmente a finales de julio. Esta situación es congruente con la hipótesis de que la abundancia vegetal (y no las condiciones climáticas directamente) constituye uno de los más importantes factores en la regulación de la cantidad poblacional del ratón doméstico. Aunque algunos autores han caracterizado al ratón doméstico como un omnívoro (Berry, 1970; Goetze et al., 1996; Stevens \& 


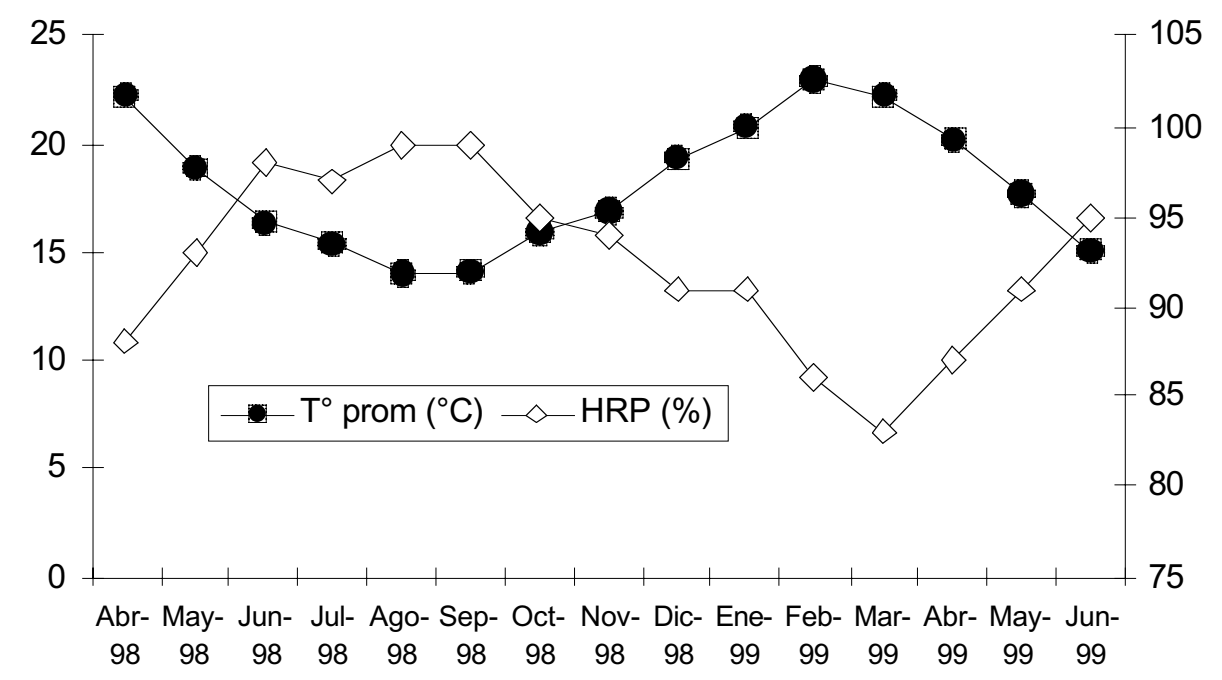

Fig. 2 - Variaciones en la temperatura promedio mensual y la humedad promedio mensual en Lachay. Datos registrados por la Estación Meteorológica de la Reserva Nacional de Lachay.

Hume, 1998), el análisis preliminar del contenido estomacal (datos no mostrados) de algunos especímenes sugiere que la población de Lachay consume frecuentemente plantas. Así, probablemente existe un relación trófica directa entre el ratón doméstico y la vegetación de estas lomas.

A partir de los resultados obtenidos en el presente trabajo no es posible explicar las causas de la mayor cantidad de ratones machos en los tres cuadrantes evaluados. Proponemos la hipótesis que los machos son más tolerantes que las hembras a las duras condiciones de las lomas durante la estación seca. Si esta idea es cierta, el número de machos y hembras debería ser equivalentes durante la estación húmeda, tal como ocurre en otros ecosistemas (Berry, 1970).

Los valores encontrados para el ámbito de hogar del ratón doméstico en Lachay son similares a los calculados para esta especie en otros ecosistemas (Quadagno, 1968; Lidicker, 1966).

Finalmente, es necesario hacer referencia al probable efecto del fenómeno "El Niño" (1997-1998) sobre los niveles de abundancia poblacional registrados en el presente trabajo. "El Niño 1997-1998" produjo un incremento en la abundancia relativa de algunas especies herbáceas, tales como Nicotiana paniculata, Nolana humifusa y Lycopersicon peruvianum en las lomas Lachay (Cano et al., 1999). Si la abundancia del ratón doméstico es directamente proporcional con la abundancia de plantas herbáceas, entonces se podría asumir que los altos niveles en la profusión del ratón doméstico (observados al inicio de la estación seca) fueron niveles anómalos y no una situación correspondiente con un año normal. Para poder entender mejor la dinámica poblacional del ratón doméstico en las lomas de Lachay, un ecosistema altamente estacional, es 
necesario realizar estudios a largo plazo (long term), intentando descubrir los patrones cíclicos que nos permitan predecir los explosivos crecimientos poblacionales que usualmente experimentan los roedores y que impactan fuertemente en la economía de muchos agricultores. Por otro lado, si la población del ratón doméstico en la lomas de Lachay es altamente sensible a los efectos del fenómeno "El Niño", constituiría un inmejorable modelo de vertebrado para estudiar los impactos biológicos de "El Niño" sobre los ecosistemas terrestres.

\section{Agradecimientos}

Los autores desean expresar su agradecimiento al Blgo. David Velarde, Jefe de la Reserva Nacional de Lachay y a todo su personal de guardaparques por las facilidades que nos brindaron durante los trabajos de campo y por el acceso a los datos climatológicos utilizados en el presente artículo. Los trabajos de campos fueron posibles gracias al apoyo logístico del NAMRID-Perú, por lo que agradecemos a su director científico, Dr. Douglas Watts.

\section{Referencias citadas}

BERRY, R., 1970 - The natural history of the house mouse. Field Study, 3: 219-262.

CANO, A., ROQUE, J., ARAKAKI, M., ARANA, C., LA TORRE, M., LLERENA, N. \& REFULIO, N., 1999 - Diversidad florística de las lomas de Lachay (Lima) durante el evento "El Niño" 1997-1998. Revista Peruana de Biología, Vol. Extraordinario: 125132.

DILLON, M., 1997 - Lomas formations-Perú. In: Centres of Plant Diversity, A Guide and Strategy for their Conservation (S. Davis, V. Heywood, O. Herrera-McBryde, J. VillaLobos \& A. Hamilton ed.): 519-527.

DIXON, K.R. \& CHAPMAN, J.A., 1980 - Harmonic mean measure of animal activity areas. Ecology, 61: 1040-1044.

FERREYRA, R., 1987 - Flora y vegetación del Perú. In: Gran Geografia del Perú. Naturaleza y Hombre (M. Mejía ed.): 63-68; Lima: Manfer.

GOETZE, J., MANNING, R., YANCEY, F. \& JONES, C., 1996 -The Mammals of Kimble County, Texas. Occasional papers, Museum of Texas Tech University: $\mathbf{N}^{\circ} \mathbf{1 6 0}$.

INRENA, 1994 - Mapa Ecológico del Perú. In: Guía Explicativa: 85-87; Lima: Instituto Nacional de Recursos Naturales.

KIE, J.G., BALDWIN J.A. \& EVAN, C. J., 1994 - CALHOME, Home Range Analysis Program, California, USA: Forest Service, Pacific Southwest Research Station.

LIDICKER, W., 1966 - Ecological observations on a feral house mouse population declining to extinction. Ecological Monographs, 36: 27-50.

MOHR, C.O., 1947 - Table of equivalent populations of North American mammals. Amererican Midland Naturalist, 37: 223-249.

QUADAGNO, D., 1968 - Home range size in feral house mice. Journal of Mammalogy, 49: 149151.

STEVENS, C. \& HUME, I., 1998 - Contributions of microbes in vertebrate gastrointestinal tract to production and conservation of nutrients. Physiological Reviews, 78: 393-427. 